\title{
Efficacy and safety of remdesivir in hospitalised COVID-19 patients: a systematic review and meta-analysis
}

\author{
Mulugeta T. Angamo ${ }^{1}$ (D) Mohammed A. Mohammed ${ }^{2} \cdot$ Gregory M. Peterson $^{1}$
}

Received: 20 April 2021 / Accepted: 21 July 2021 / Published online: 31 July 2021

(c) Crown 2021

\begin{abstract}
Purpose This review was aimed to synthesise the best available evidence on the effectiveness and safety of remdesivir in the treatment of moderate to severe COVID-19.

Method Randomised controlled trials (RCTs) and observational studies reporting the effectiveness and safety of remdesivir were searched via databases and other sources from December 2019 to December 2020. Two independent reviewers performed literature screening, data extraction and assessment of risk bias. Seven studies involving 3686 patients were included. Results Treatment with remdesivir was associated with an increase in clinical recovery rate by $21 \%$ (RR $1.21 ; 95 \%$ CI $1.08-1.35$ ) on day 7 and $29 \%$ (RR $1.29 ; 95 \%$ CI 1.22-1.37) on day 14 . The likelihoods of requiring high-flow supplemental oxygen and invasive mechanical ventilation in the remdesivir group were lower than in the placebo group by $27 \%$ (RR 0.73 ; 95\% CI 0.54-0.99) and 47\% (RR 0.53; 95\% CI 0.39-0.72), respectively. Remdesivir-treated patients showed a 39\% (RR $0.61 ; 95 \%$ CI $0.46-0.79)$ reduction in the risk of mortality on day 14 compared to the control group; however, there was no significant difference on day 28. Serious adverse effects (SAEs) were significantly less common in patients treated with remdesivir, with an absolute risk difference of $6 \%$ (RD -0.06 ; $95 \% \mathrm{CI}-0.09$ to -0.03 ).

Conclusion Despite conditional recommendation against its use, remdesivir could still be effective in early clinical improvement; reduction of early mortality and avoiding high-flow supplemental oxygen and invasive mechanical ventilation among hospitalised COVID-19 patients. Remdesivir was also well tolerated without significant SAEs compared to placebo, yet available evidence from clinical studies support the need to conduct close monitoring.
\end{abstract}

Keywords Remdesivir $\cdot$ Coronavirus deisease-19 $\cdot$ Safety $\cdot$ Effectiveness

Mulugeta T. Angamo

Mulugeta.Angamo@utas.edu.au

1 School of Pharmacy and Pharmacology, University of Tasmania, Hobart, Australia

2 School of Pharmacy, The University of Auckland, Auckland, New Zealand 


\section{Key Points}

This systematic review and meta-analysis pooled data from 7 studies involving a total of 3686 patients hospitalised with moderate-to-severe COVID-19 and compared the effectiveness and safety of remdesivir with placebo or standard treatment.

Although treatment with remdesivir was associated with an increase in the early clinical recovery rate, reduction in the risk of early mortality and a lower likelihood of requiring high-flow supplemental oxygen and invasive mechanical ventilation compared to the placebo or standard care, there was no significant difference in reducing mortality on day 28 for patients with COVID- 19 .

Remdesivir-treated patients also showed less common serious adverse effects than patients treated with the placebo or standard care.

\section{Introduction}

Worldwide, over 131 million people have been diagnosed with Coronavirus Disease 2019 (COVID-19), resulting in nearly 2.9 million deaths [1]. As a global pandemic, it has resulted in a profound, negative impact on the healthcare system, economy and financial markets due to the public health crisis, loss of life, reduced productivity, business closures, trade disruption, and devastation of the tourism industry [2-4]. Healthcare systems worldwide are now experiencing varying levels of stress amid infection rates that require a collective response [5].

Several therapeutic agents have been evaluated for the treatment of COVID-19 in an attempt to control viral replication [6]. These involve various approaches, such as the antiviral repurposing of drugs that were used in severe acute respiratory syndrome (SARS)-CoV-1 and MERS (Middle East respiratory syndrome)-CoV, including antiretroviral agents; using immunoglobulins and convalescent plasma; and the bioinformatics screening of chemical libraries for existing compounds/drugs that are likely to act on SARSCoV-2 [7]. Multiple antiviral treatment options are under investigation for COVID-19 infections [8], including remdesivir, which was first developed for the treatment of Ebola infection. Remdesivir is a nucleotide prodrug that is metabolised intracellularly to the active nucleoside triphosphate (ATP) and interferes with viral RNA-dependent RNA polymerase activity [7]. This activity led to its use in patients with SARS-CoV-2 infection (COVID-19), in the absence of any effective treatment. However, the pharmacology and pharmacokinetics of remdesivir within the respiratory tract and other infected organs of critically ill patients with COVID-19 remain largely unknown. Safety information is currently fragmented and limited [8].

A small number of observational and experimental studies have been conducted to examine the clinical benefits and safety of remdesivir [9-11]. The first randomised, placebocontrolled clinical trial in Wuhan, China, could not complete enrolment of the study participants to meaningfully evaluate the efficacy of the drug [10]. A preliminary report by Gillenwater et al. [12] and a final report by Beigel et al. [9] of a larger randomised, double-blind placebo-controlled clinical trial of a 10-day course of remdesivir revealed a significantly shorter time to recovery when compared with placebo (a median of 11 days vs 15 days). Subsequently, a randomised, open-label, multicentre study compared 5-day and 10-day courses of remdesivir with standard care. There was a significant difference in clinical status between standard care and a 5-day course, while a 10-day course did not show statistically significant difference in clinical status [13]. Another randomised, open-label trial that compared outcomes of 5-day versus 10-day courses of remdesivir in patients requiring oxygen therapy showed no significant difference in clinical status [11]. These findings, although conflicting, prompted the US Food and Drug Administration (FDA) to grant emergency use authorisation of remdesivir for patients with severe COVID-19 [14] and the European Medicines Agency (EMA) [15] to grant conditional marketing authorisation of remdesivir for the treatment of COVID19 in patients aged 12 years or older.

Although there appears to be a favourable benefit-risk profile for remdesivir compared with placebo or standard care, there is still limited information on its efficacy and safety [16] to inform practitioners, policymakers and leaders in formulating management guidelines and to provide directions for future research. The findings of existing systematic reviews and meta-analysis are conflicting [17-24]. Despite clinical improvements and reduction in mortality [17, 20,21, 24-27], the World Health Organisation has recently issued a conditional recommendation against its use in hospitalised patients, regardless of disease severity. This prompted us to conduct an updated further systematic review and meta-analysis, with the aim of synthesising the best available evidence on the efficacy and safety of remdesivir in the treatment of moderate-to-severe COVID-19. 


\section{Method}

\section{Criteria for considering studies for this review}

\section{Types of studies}

The study designs considered were both experimental and quasi-experimental, including randomised controlled trials (RCTs), controlled clinical trials and experimental studies where randomisation had been used. A comparative study without randomisation and a cohort study were also included based on the outcome measures and reviewers' agreement. Studies assessing the outcomes in patients with co-infection with hepatitis-B or human-immunodeficient virus (HIV) were excluded.

\section{Types of participants}

This review included hospitalised patients aged 12 years or over with moderate-to-severe COVID-19 and being treated with remdesivir and placebo or standard care. Patients randomised to a placebo group took matching normal saline and those in the standard care group were more commonly prescribed other agents with putative activity against COVID19 including corticosteroids, hydroxychloroquine/chloroquine, lopinavir-ritonavir, tocilizumab and azithromycin.

\section{Type of intervention and comparators}

This review compared remdesivir (5-day and /or 10-day course) as an intervention with a 5- or 10-day course of placebo or standard care.

\section{Types of outcome measures}

The primary outcomes were median time to recovery; rates of clinical recovery on day 7,14 and 28; all-cause mortality on day 14 and 28; and the requirement for supplemental oxygen and mechanical ventilation on day 7, 14 and 28 . The secondary outcome was remdesivir safety indicated by serious adverse effects, grade 3 (severe) and grade 4 (lifethreatening) adverse effects and discontinuation of treatment due to adverse effects during the follow-up period. Serious adverse effects included respiratory failure or acute respiratory distress syndrome, cardiopulmonary failure, pulmonary embolism, recurrence of COVID-19, cardiac arrest, septic shock, acute kidney injury, and diabetic ketoacidosis [10]. Recovery was defined as hospital discharge or need for hospitalisation but without supplemental oxygen or ongoing medical care.

\section{Search strategies}

This review was conducted using a Preferred Reporting Items for Systematic reviews and Meta-analyses (PRISMA) reporting guidelines. A comprehensive search was performed on MEDLINE, WEB OF SCIENCE, Cochrane Central Register of Controlled Trials (CENTRAL), Cumulative Index to Nursing and Allied Health Literature (CINAHL), GOOGLE SCHOLAR, PROQUEST Central, EMBASE using a combination of medical subject headings (MeSH), and free-text terms until 31 December 2020. Searching was conducted by each engine using the keywords: remdesivir AND (COVID-19 OR coronavirus OR coronavirus disease OR coronavirus disease-19 OR severe acute respiratory syndrome OR SARS-CoV-2) with no limitations for time or language. We repeated the search strategy using the above combination but replacing remdesivir with "adenosine nucleoside triphosphate analog" and "Veklury".

\section{Data collection and analysis}

\section{Selection of studies}

Two authors (MA and MM) independently reviewed the titles and abstracts for eligibility according to the inclusion and exclusion criteria. Other reviewer (GP) randomly assessed the full-text of some articles. Discrepancies were resolved by consensus. If the two reviewers were unable to reach consensus, they consulted a senior reviewer (GP).

\section{Data extraction and management}

The data extracted included mean/median age, country of study, total number of patients included, outcomes, coexisting illnesses, the difference in the requirement of supplemental oxygen and ventilation between intervention and control group, the primary outcomes and the secondary outcomes.

\section{Assessment of risk of bias and publication bias in included studies}

The risk of bias for randomised controlled trial studies was assessed by two independent reviewers (MA and MM) using a modified version of the Cochrane Risk of Bias tool [28]. Consensus were made with other author (GP) with discussion on the report. The risk of bias tool consists of six domains: selection bias, reporting bias, performance bias, detection bias, attrition bias and other sources of bias [28]. The risk of bias for non-randomised studies was assessed 
using the risk of bias in nonrandomised studies of interventions (ROBINS-I) tool [29]. The publication bias was not assessed as there were less than ten studies involved in the analysis [30].

\section{Measures of treatment effect}

The pooled effect estimate of the dichotomous outcomes were presented using a fixed-effect model. As per the Cochrane Review Manager recommendation, when there was heterogeneity that could not readily be explained, a random effect model was used.

\section{Data analysis}

Review Manager version 5.4 (The Cochrane Collaboration, Copenhagen) software was used for the statistical analysis. Meta-analysis was undertaken for the time to recovery, clinical recovery, all-cause mortality, requirement for supplemental oxygen and ventilation, serious adverse effects, discontinuation of remdesivir due to adverse effects and grade 3 or 4 adverse effects. Risk ratio (RR) with $95 \%$ confidence interval (CI) was used to summarise the efficacy of remdesivir, while rate difference (RD) with 95\% CI and risk ratio (RR) with $95 \%(\mathrm{CI})$ were used to summarise the safety of remdesivir.

\section{Assessment of heterogeneity}

The heterogeneity between studies was assessed using a Chi-squared and/or tau squared $\left(I^{2}\right)$ test. $I^{2}$ values were considered as moderate for $30-60 \%$, substantial for $50-90 \%$ and considerable for $75-100 \%$ of heterogeneity between studies [30]. In case of an $I^{2}$ value $>50 \%$, the potential sources of heterogeneity attributed to severity of the disease, severity of the adverse effect, level of oxygen requirement were explored through subgroup analyses [31]. All statistical tests were carried out with the Review Manager (RevMan) software suite version 5.4 (The Cochrane Collaboration, Copenhagen) [28]. Statistical significance was defined as a $p$ value $<0.05$.

\section{Results}

\section{Characteristics of the included studies}

We identified a total of 1186 records from electronic database searches and other sources. After removal of duplicates, 659 studies were screened by title and abstracts. After a thorough full-text review of 17 studies, 4 randomised clinical trials [9-11, 13] and 3 non-randomised observational studies [5, 32, 33] with a total number of study participants of 3686 (1553 patients on 10-day remdesivir vs 391 patients on 5-day remdesivir vs 1742 patients on placebo or standard treatment) were included in the systematic review and meta-analysis (Fig. 1). Of the four randomised trials, three were placebo-controlled $[9,10,13]$ and one trial compared 5 -and 10-day remdesivir treatments [11]. Ten studies were excluded due to lack of comparator group and clear outcome measuring parameters. In all included studies, the intervention group (treatment arm) consistently received a loading dose of remdesivir of $200 \mathrm{mg}$ on day 1 followed by 4 or 9 days of $100 \mathrm{mg}$ once daily in the 5-day or 10-day arms, respectively (Table 1).

In all studies, more males than females were included in both the remdesivir and control groups. Diabetes and hypertension were the most common comorbidities in both the remdesivir and placebo groups. Nearly half (46\%) of the patients in Kalligeros et al. [32] study were obese (Table 1).

According to six/seven-point ordinal scale, most patients in Wang's study [10] $(231 / 236,97.9 \%)$ and Goldman's study [11] (329/397, 82.9\%) were on low or high-flow supplemental oxygen. Only $(93 / 584,16 \%)$ of the patients in Spinner's study [13] required high- and low-flow supplemental oxygen while most $(491 / 584,84 \%)$ patients did not require supplemental oxygen. In the Beigel et al. study [9], the majority of the patients $(618,60.8 \%)$ required either low- or high-flow supplemental oxygen (421 low-flow oxygen, 197 high-flow oxygen) and about one-third (272, 26.7\%) required invasive mechanical ventilation/extracorporeal membrane oxygenation (ECMO) (Table 1).

\section{Efficacy outcome measures}

\section{Clinical recovery}

The findings in relation to clinical recovery were pooled from three clinical trials $[9,10,13]$ and one observational study [32] using a fixed effect model. According to these studies, treatment with remdesivir was associated with a statistically significant $21 \%$ increase in recovery rate (RR $1.21 ; 95 \%$ CI 1.08-1.35) relative to the control group on day 7. Similarly, treatment with remdesivir was associated with a $29 \%$ increase in clinical recovery rate (RR 1.29; $95 \%$ CI 1.22-1.37) on day 14. However, its effect declined to 9\% (RR 1.09; 95\% CI 1.04-1.14) on day 28. Heterogeneity was not significant for days 7 and 28; however, the study of Beigel et al. [9] on day-14 with its high effect size resulted in significant heterogeneity $\left(p=0.002 ; I^{2}=76 \%\right)$. After removing Beigel et al. from the analysis, the remaining studies showed a $20 \%$ increase in recovery rate (RR 1.20; $95 \%$ CI 1.12-1.27) with mild heterogeneity $\left(p=0.42 ; I^{2}=0 \%\right)$ (Fig. 2). 
Fig. 1 PRISMA flow diagram of the included studies

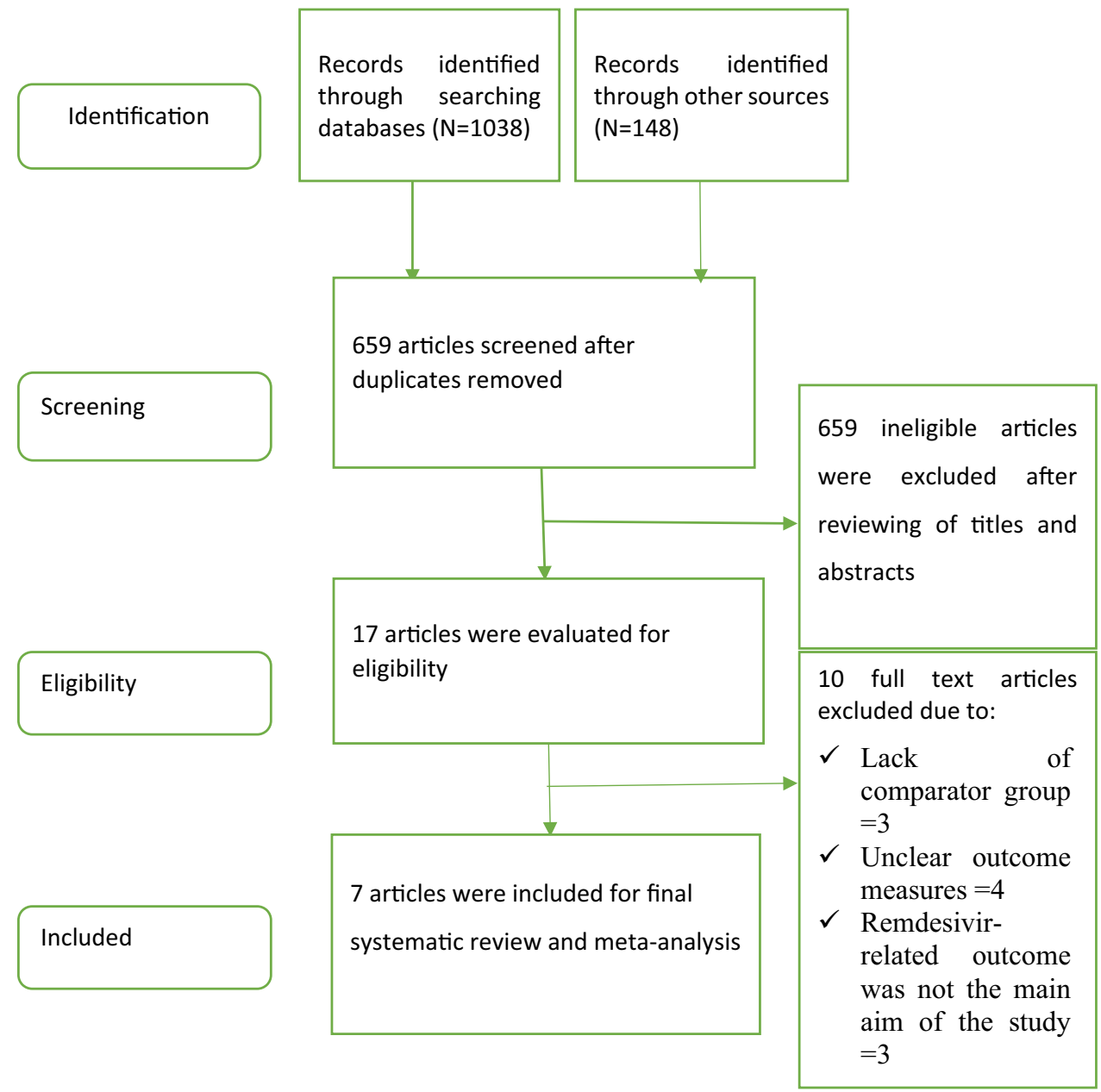

\section{Requirement of supplemental oxygen and ventilation}

The pooled analysis from three RCTs $[9,10,13]$ showed no significant difference between patients treated with remdesivir and placebo or standard care in the requirement for low-flow oxygen (RR 0.76; 95\% CI 0.55-1.06, $p=0.11$ ). However, the likelihood of requiring high-flow supplemental oxygen was $27 \%$ lower in remdesivir-treated patients than placebo (RR 0.73 ; 95\% CI $0.54-0.99, p=0.04$ ). The likelihood of requiring invasive mechanical ventilation or ECMO in the remdesivir group was $47 \%$ lower than the placebo group (RR 0.53; 95\% CI 0.39-0.72, $p<0.001$ ) (Fig. 3). Subgroup analysis comparing days 7, 14 and 28 showed that remdesivir-treated patients were less likely to require low- or high-flow oxygen and mechanical ventilation than placebotreated patients (RR 0.83 ; 95\% CI $0.77-0.90, p<0.001$ ) (Annex II).

\section{All-cause mortality}

Pooled estimate for all-cause mortality were determined from four studies [9, 10, 13, 32]. Remdesivir-treated patients showed a $39 \%$ reduction in the risk of mortality on day 14 compared to the control (placebo) group (RR $0.61 ; 95 \% \mathrm{CI}$ $0.46-0.79, p=0.003$ ). However, there was no significant difference between remdesivir and placebo (RR 0.78; 95\% CI $0.59-1.03, p=0.09$ ) on day 28 . When the study by Wang et al. was removed (using sensitivity analysis to see the effect measure), the pooled data showed a significant difference between remdesivir and placebo group on day 28 (RR 0.73 , $95 \%$ CI $0.54-0.99, p=0.04$ ) with a $27 \%$ lower risk of mortality in remdesivir group with mild heterogeneity ( $p=0.92$; $I^{2}=0 \%$ ) (Fig. 4).

\section{Median time to recovery and median time to discharge}

The findings related to median time to recovery were pooled from three studies $[9,10,13]$. Treatment with remdesivir was associated with a $37 \%$ reduction in a median time to recovery compared to a placebo or standard care (RR 0.63; 95\% CI $0.43-0.92 ; p=0.02$ ) with mild heterogeneity. Similarly, pooled data from two studies $[9,10]$ showed that treatment with remdesivir was associated with a $46 \%$ reduction 


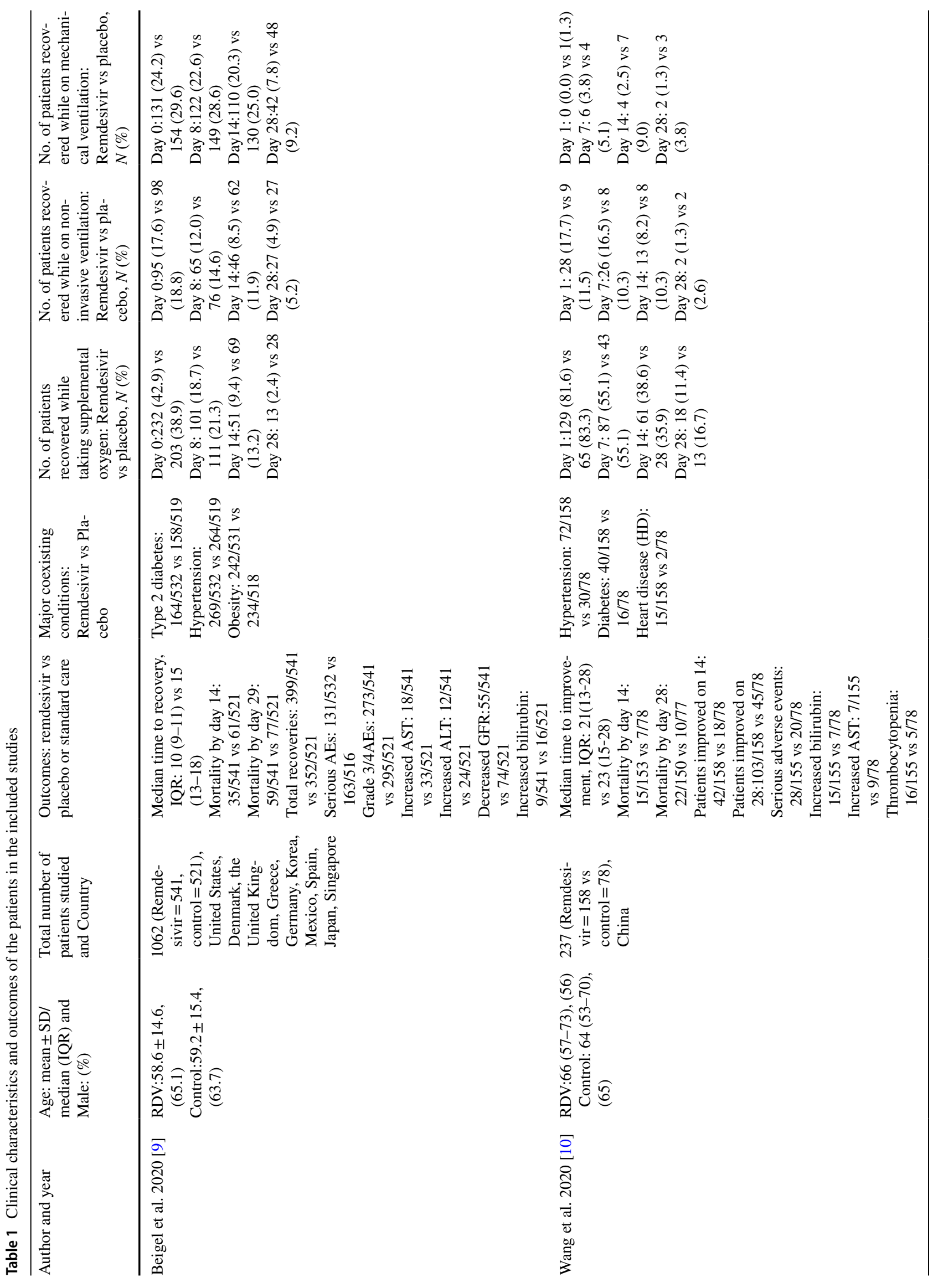




\begin{tabular}{|c|c|c|}
\hline 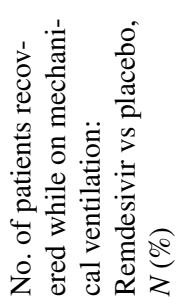 & 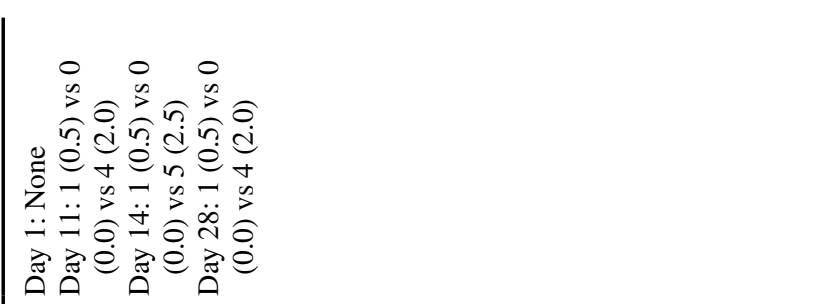 & 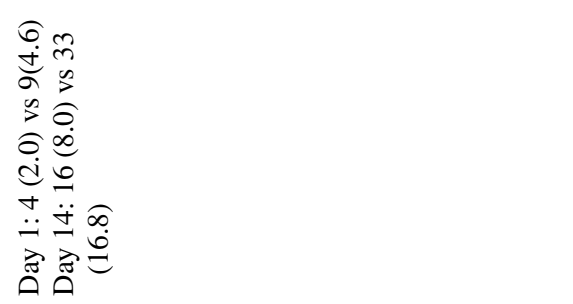 \\
\hline 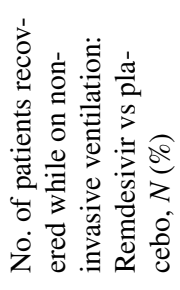 & 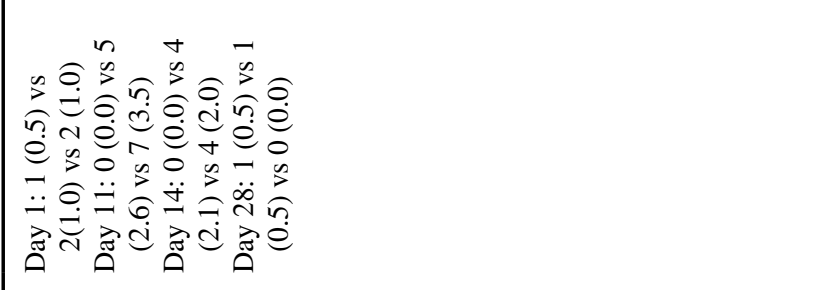 & 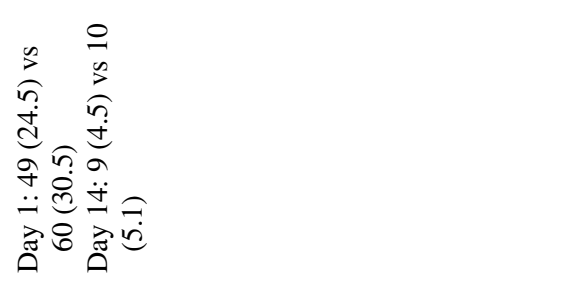 \\
\hline 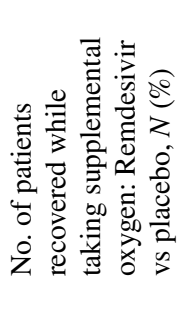 & 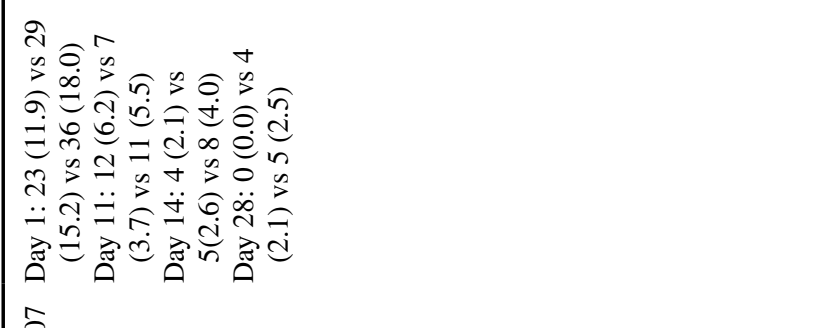 & 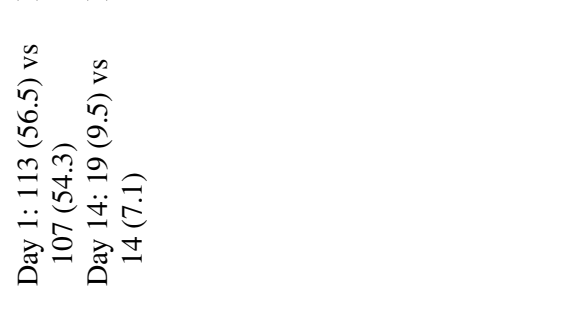 \\
\hline 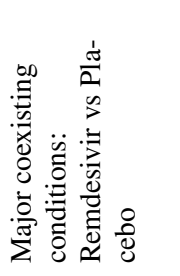 & 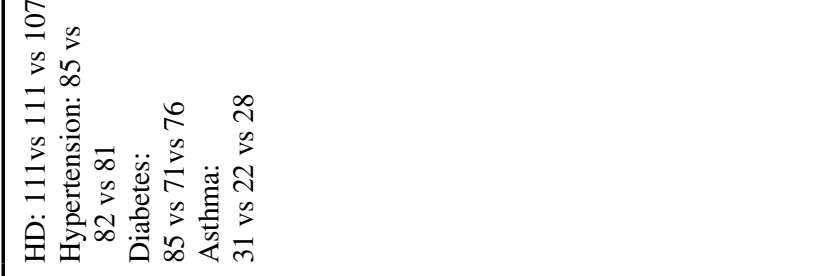 & 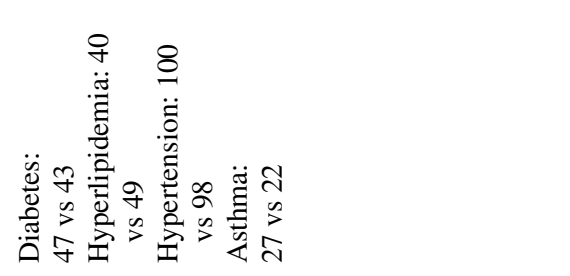 \\
\hline 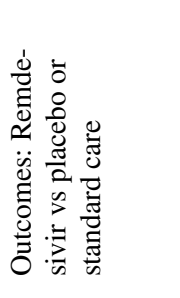 & 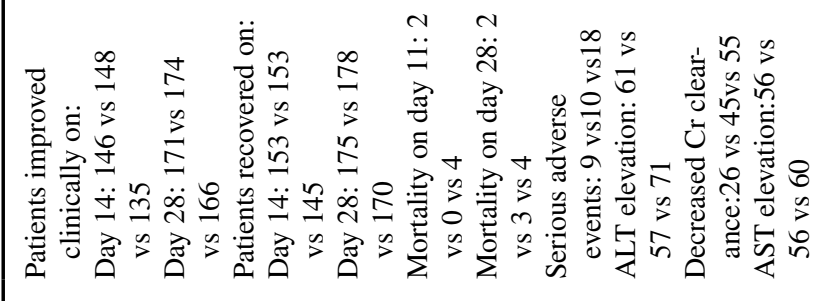 & 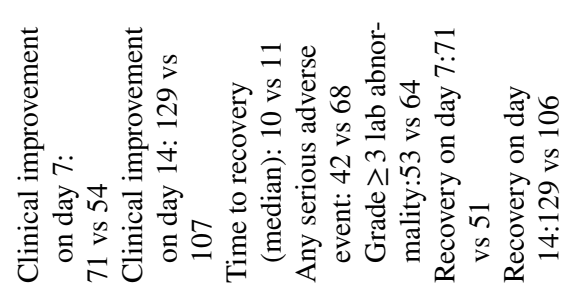 \\
\hline 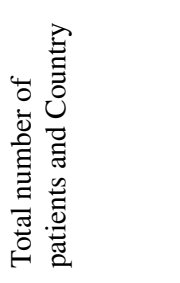 & 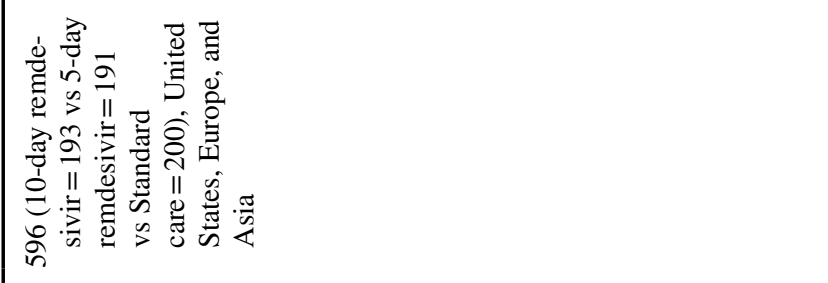 & 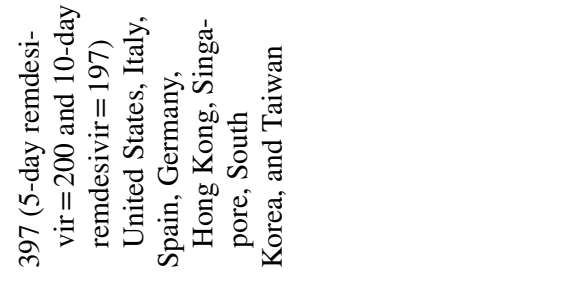 \\
\hline 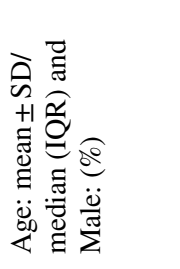 & 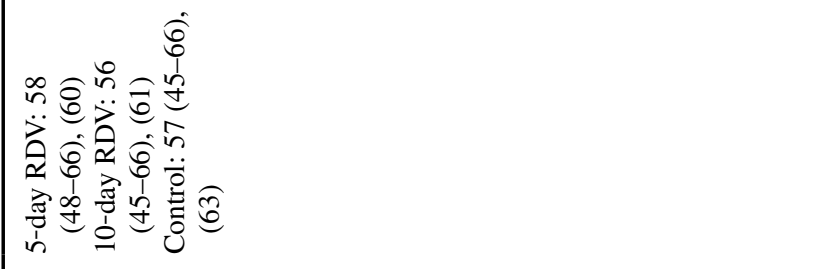 & 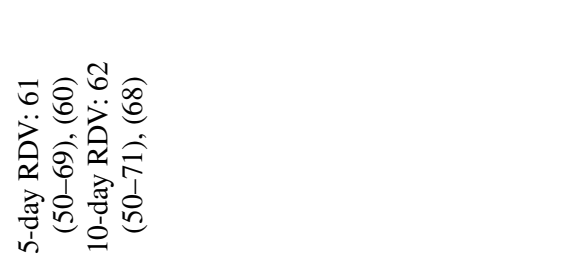 \\
\hline & 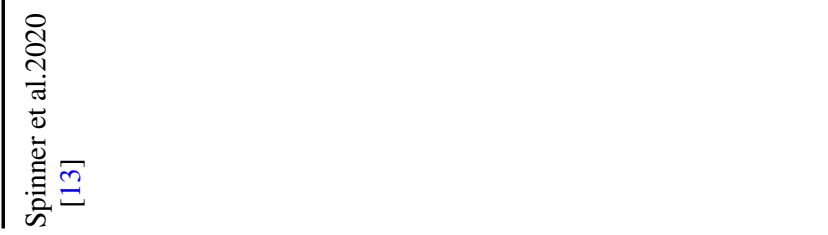 & 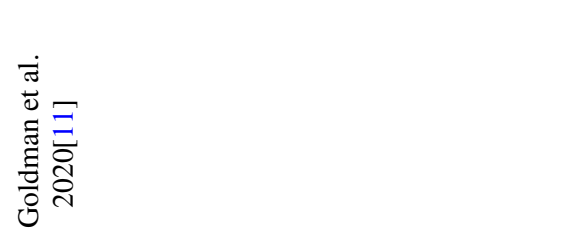 \\
\hline
\end{tabular}




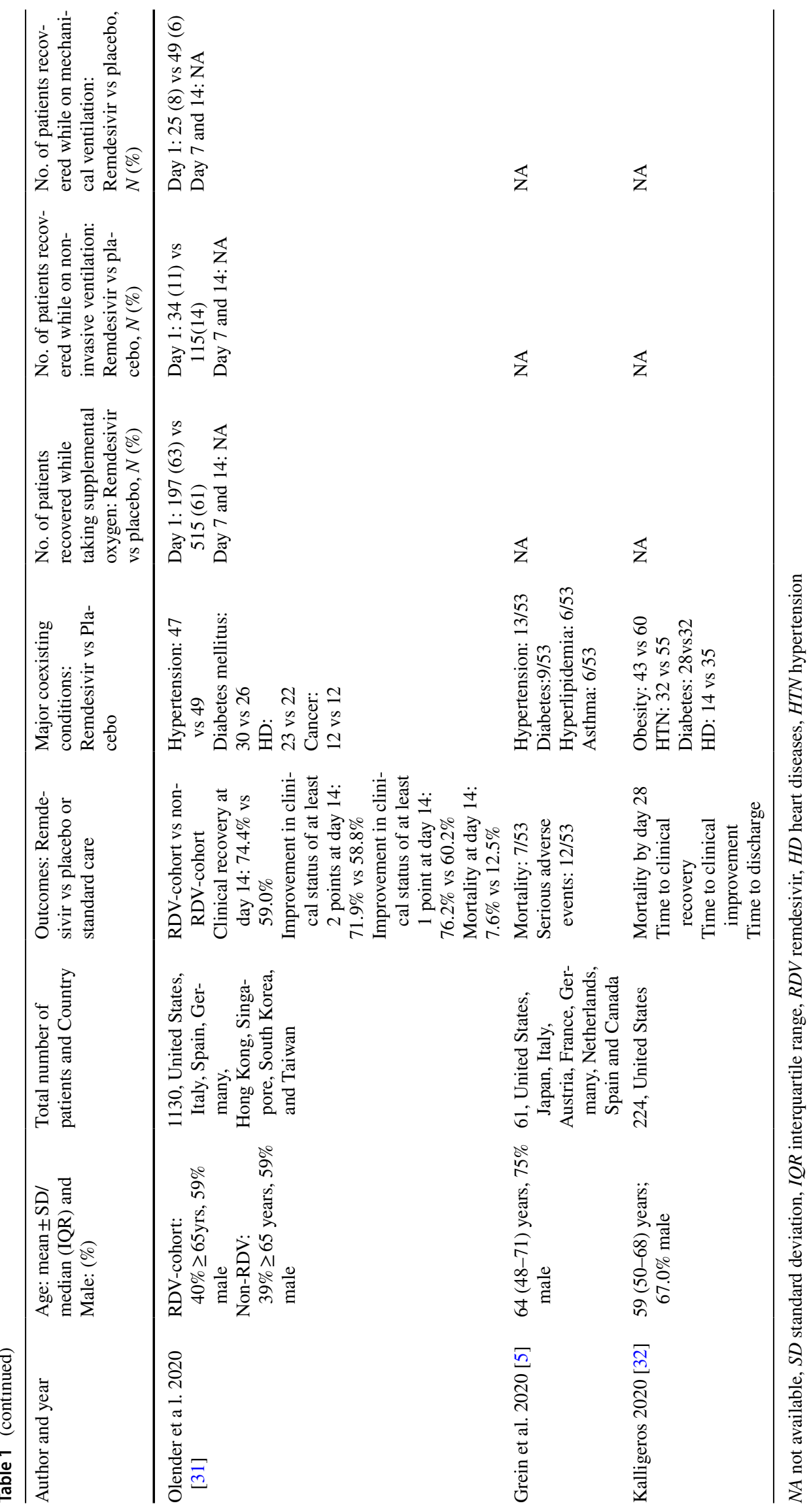


Number of patients with clinical recovery on day 7

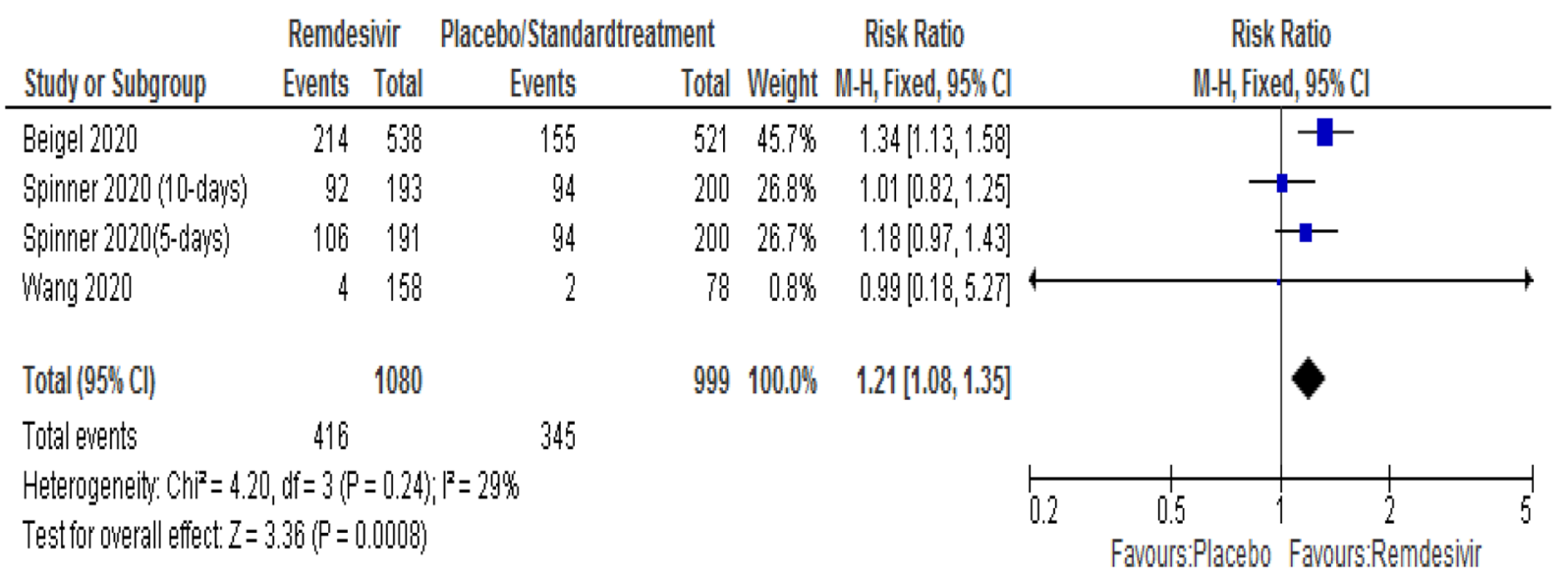

Number of patients with clinical recovery on day 14

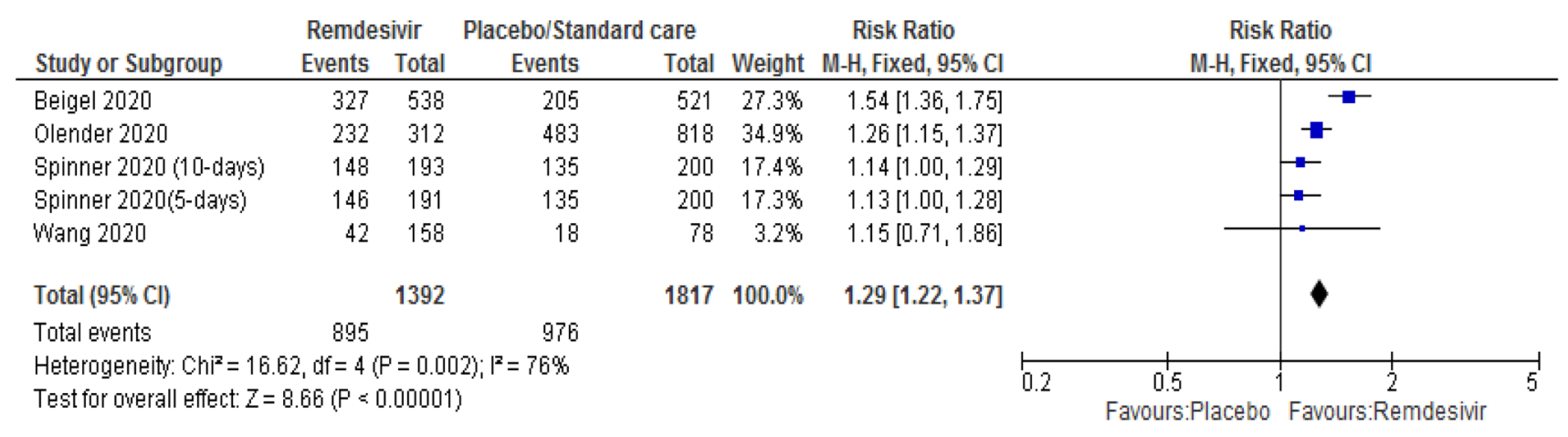

Number of patients with clinical recovery on day 28

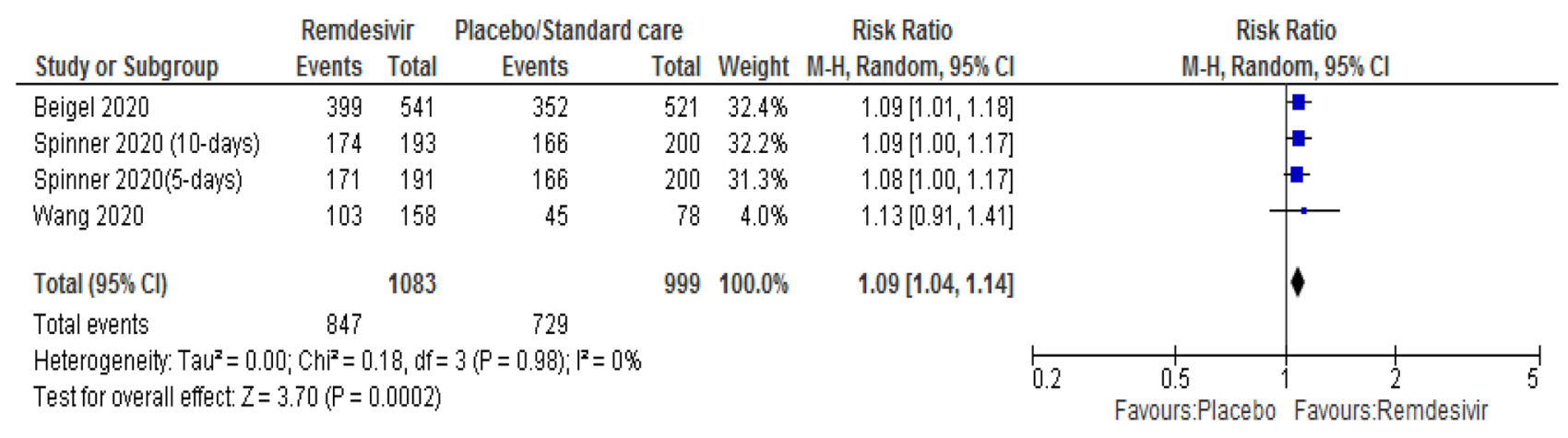

Fig. 2 Forest plot of clinical recovery rates on day 7, 14 and 28

in median time to discharge from hospital compared to placebo (RR 0.54; 95\% CI 0.34-0.86; $p=0.007$ ) (Fig. 5).

\section{Safety outcomes}

The pooled data from three RCTs $[9,10,13]$ and two observational studies $[5,32]$ on the safety outcomes of remdesivir showed that serious adverse effects were significantly less common in patients treated with remdesivir compared to placebo with an absolute risk difference of $6 \%(\mathrm{RD}-0.06$; $95 \% \mathrm{CI}-0.09$ to $-0.03 ; p<0.001)$. However, there was no significant difference between remdesivir- and placebotreated patients in discontinuing treatment due to adverse effects (RD $-0.00,95 \% \mathrm{CI}-0.03$ to $0.02 ; p=0.80$ ) with considerable heterogeneity $\left(I^{2}=85 \%\right)$. Removing the study by Beigel et al. [9] showed an increased risk of discontinuing 
Number of patients receiving low-flow supplemental oxygen

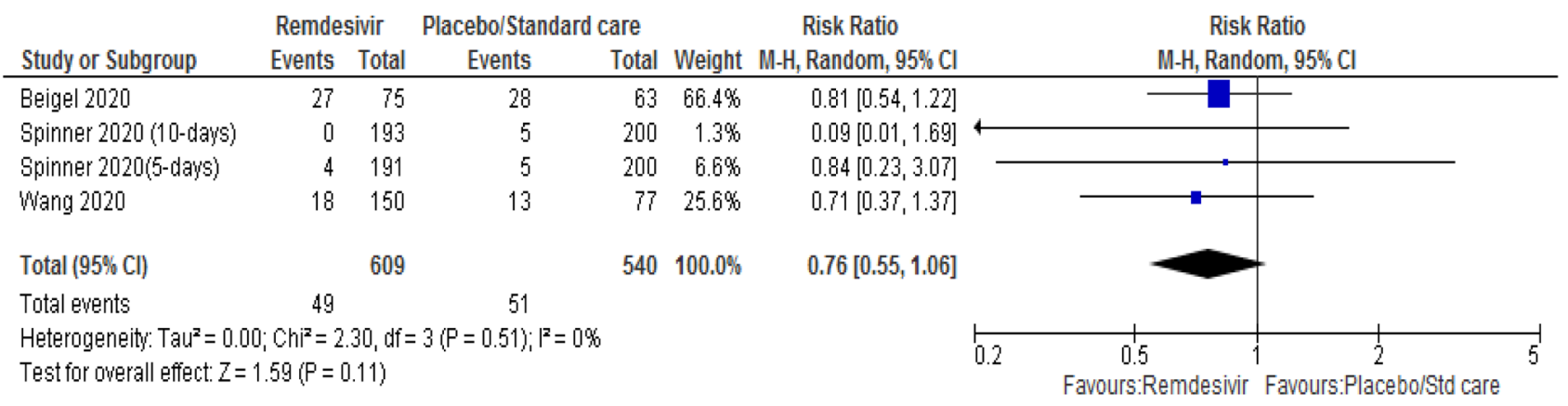

Number of patients receiving non-invasive ventilation/high-flow oxygen

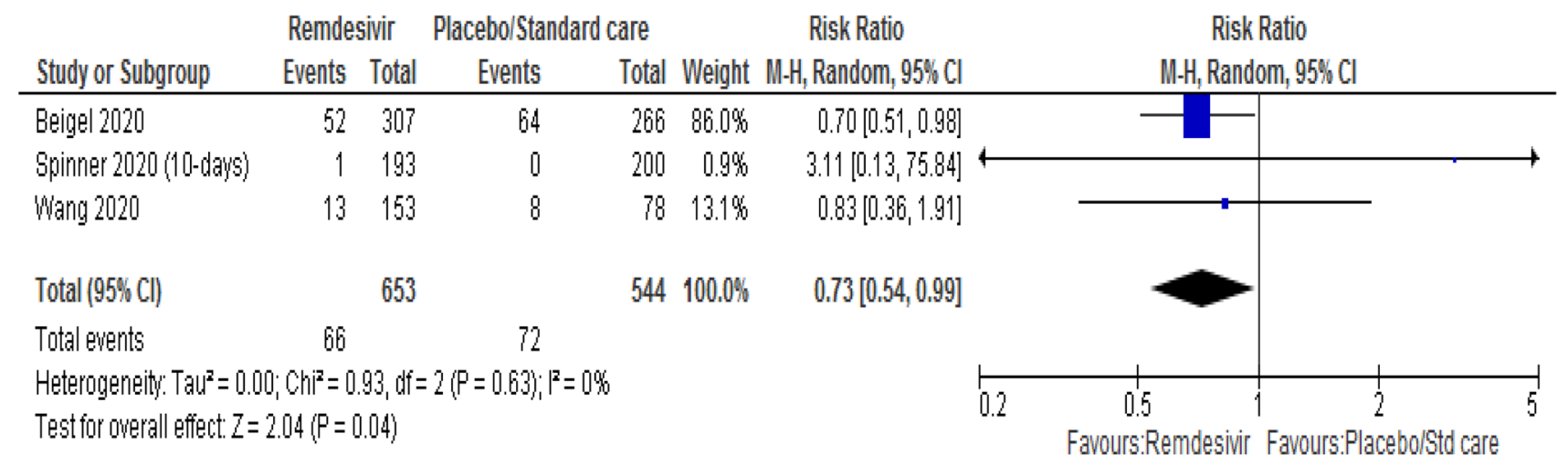

Number of patients requiring mechanical ventilation or ECMO

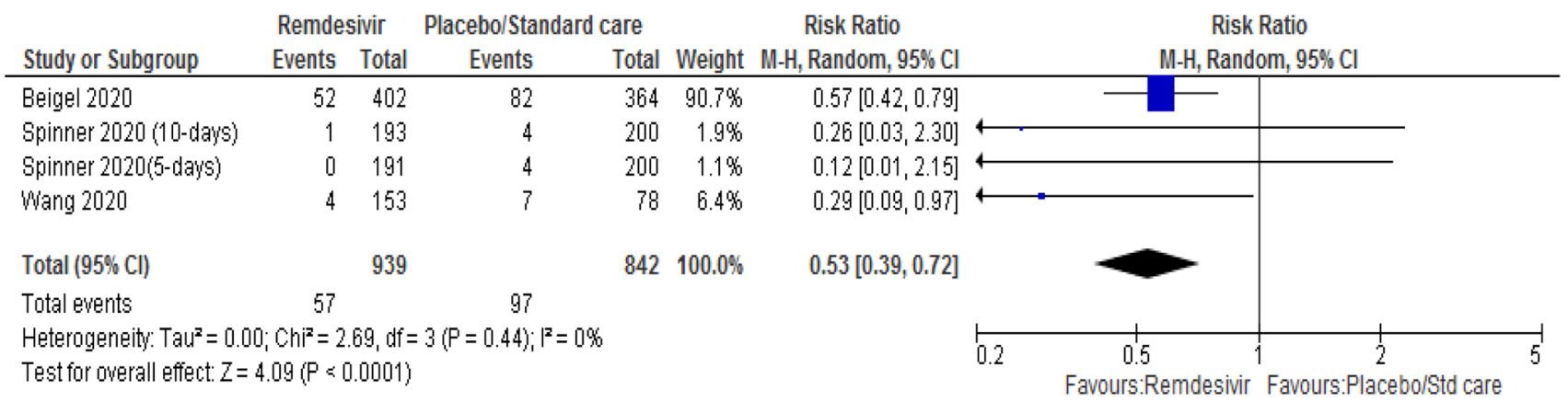

Fig. 3 Forest plot of the requirement of supplemental oxygen and ventilation

treatment due to adverse effects in the placebo group compared to remdesivir-treated patients (RD 0.04; 95\% CI $0.02-0.06 ; p<0.001)$ with moderate heterogeneity ( $\mathrm{p}<$ $0.01 ; \mathrm{I}^{2}=30 \%$ ). Treatment with remdesivir was associated with a $26 \%$ lower risk of presenting with grade 3 (severe) and grade 4 (life-threatening) adverse effects (RR 0.74; $95 \%$ CI 57-97; $p=0.03$ ) compared to placebo. Similarly, remdesivir was associated with a $24 \%$ lower risk of AST elevation (RR 0.76; 95\% CI 0.60-0.96; $p=0.02$ ) with significant heterogeneity $\left(I^{2}=71 \%\right)$ compared with placebo. Removing the observational study by Kalligeros et al. [33] improved the heterogeneity $\left(I^{2}=46 \%\right)$ with a lower risk of AST elevation in the remdesivir group (RR 0.58; 95\% CI 0.43-0.79, $p<0.001$ ). There was also a lower risk of ALT elevation (RR $0.80 ; 95 \%$ CI $0.69-0.94 ; p=0.006)$ and renal function (RR 0.66 ; 95\% CI $0.55-0.80 ; p<0.001)$ in the remdesivir group compared to standard treatment or placebo (Fig. 6). 
All-cause mortality on day 14

\begin{tabular}{|c|c|c|c|c|c|c|c|c|c|}
\hline \multirow[b]{2}{*}{ Study or Subgroup } & \multicolumn{2}{|c|}{ Remdesivir } & \multicolumn{2}{|c|}{ Placebo/Standard care } & \multicolumn{2}{|c|}{ Risk Ratio } & \multirow{2}{*}{\multicolumn{3}{|c|}{$\begin{array}{c}\text { Risk Ratio } \\
\text { M-H, Fixed, } 95 \% \mathrm{Cl}\end{array}$}} \\
\hline & Events & Total & Events & Total & Weight & M-H, Fixed, $95 \% \mathrm{Cl}$ & & & \\
\hline Beigel 2020 & 35 & 541 & 61 & 521 & $45.8 \%$ & $0.55[0.37,0.82]$ & 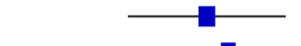 & & \\
\hline Olender 2020 & 24 & 312 & 102 & 818 & $41.5 \%$ & $0.62[0.40,0.94]$ & & & \\
\hline Spinner 2020 (10-days) & 2 & 193 & 4 & 200 & $2.9 \%$ & $0.52[0.10,2.80]$ & $\longleftarrow$ & & \\
\hline Spinner 2020(5-days) & 1 & 191 & 4 & 200 & $2.9 \%$ & $0.26[0.03,2.32]$ & $\longleftarrow$ & & \\
\hline Wang 2020 & 15 & 153 & 7 & 78 & $6.8 \%$ & $1.09[0.46,2.57]$ & & & \\
\hline Total (95\% Cl) & & 1390 & & 1817 & $100.0 \%$ & $0.61[0.46,0.79]$ & & & \\
\hline Total events & 77 & & 178 & & & & & & \\
\hline $\begin{array}{l}\text { Heterogeneity: } \mathrm{Ch}^{2}=2.6 \\
\text { Test for overall effect: } Z=\end{array}$ & $\begin{array}{l}\mathrm{df}=4(\mathrm{P} \\
65(\mathrm{P}=0\end{array}$ & $\begin{array}{l}=0.62) ; \\
.0003)\end{array}$ & $i^{2}=0 \%$ & & & & $\begin{array}{c}0.5 \\
\text { Favours:Remdesiv }\end{array}$ & ${ }^{1}$ ir Favours: Pl & $2^{2}$ lacebo/std care \\
\hline
\end{tabular}

All-cause mortality on day 28

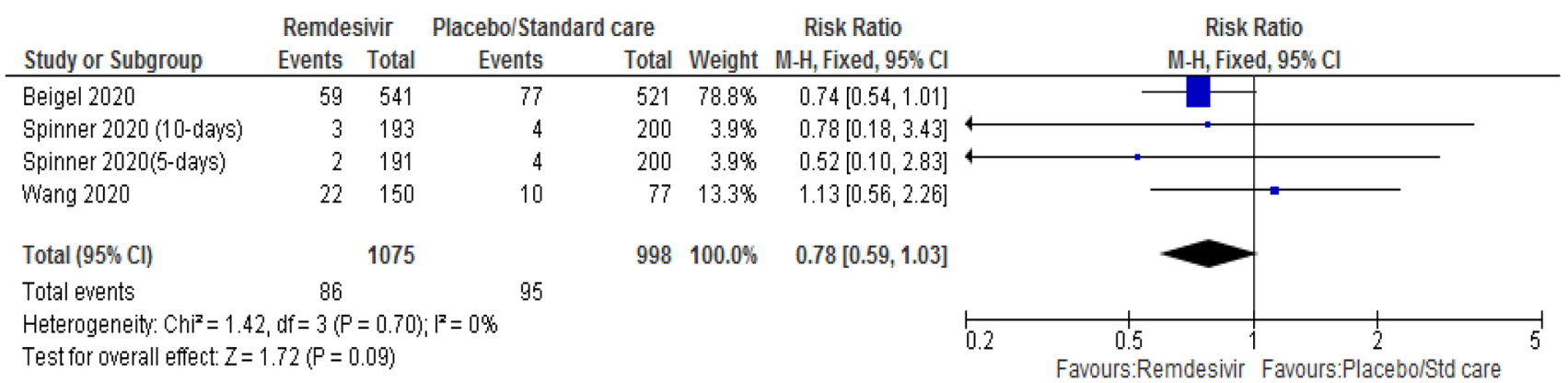

Fig. 4 Forest plot of all-cause mortality on day 14 and 28

\section{Discussion}

This systematic review and meta-analysis aimed to provide comprehensive evidence regarding the clinical benefits and safety profile of remdesivir in hospitalised patients with COVID-19. We used a thorough approach to search for the best available data and provided a summary of evidence pooled from 7 studies with a total of 3686 patients with moderate-to-severe COVID-19. Treatment with remdesivir was associated with an increase in the early clinical recovery rate, reduction in the risk of early mortality and a lower likelihood of requiring high-flow supplemental oxygen and invasive mechanical ventilation, compared to the placebo or standard care. Remdesivir-treated patients also showed less common serious adverse effects than patients treated with the placebo or standard care.

We found that treatment with remdesivir was associated with an increased clinical recovery rate by $21 \%, 29 \%$ and $9 \%$ on days 7,14 and 28 , respectively, relative to the placebo or standard care group. After removing Beigel et al. [9] on day 14 , which had a greater effect size resulting in considerable heterogeneity, the pooled result showed that treatment with remdesivir increased the clinical recovery rate by $20 \%$ compared to placebo (RR 1.20; 95\% CI 1.12-1.27). Likewise, the recovery rate with remdesivir treatment decreased from $29 \%$ on day $14-9 \%$ on day 28 , suggesting that remdesivir could be more effective for early treatment among hospitalised COVID-19 patients. A network meta-analysis by Jiang et al. [25] showed that both 10-day and 5-day remdesivir regimens were associated with higher probabilities of clinical recovery (RR of 10-day regimen: $1.24 ; 95 \%$ CI 1.07-1.43, RR of 5-day regimen: 1.47 ; 95\% CI 1.16-1.87) compared with placebo. Another network meta-analysis of four RCTs by Yokoyama et al. [24] demonstrated a higher clinical improvement rate in both 5-day (OR 1.89; 95\% CI 1.40-2.56) and 10-day (OR 1.38; 95\% CI 1.15-1.66) remdesivir groups compared to a standard care group. A recent systematic reviews and meta-analysis of RCTs [17, 20, 26] reported a consistent clinical benefit of remdesivir in comparison to a placebo or standard care.

Although there was no significant difference between remdesivir and placebo group with respect to the need for low-flow supplemental oxygen (RR 0.76 ; 95\% CI 0.55-1.06), remdesivir-treated patients were less likely to require high-flow supplemental oxygen (RR 0.73; 95\% CI 0.54-0.99) or invasive mechanical ventilation or ECMO (RR $0.53 ; 95 \%$ CI $0.39-0.72$ ) than patients treated with placebo. A subgroup analysis for days 7, 14 and 28 also showed that remdesivir-treated patients were less likely to require highflow oxygen and invasive mechanical ventilation (RR 0.83; 


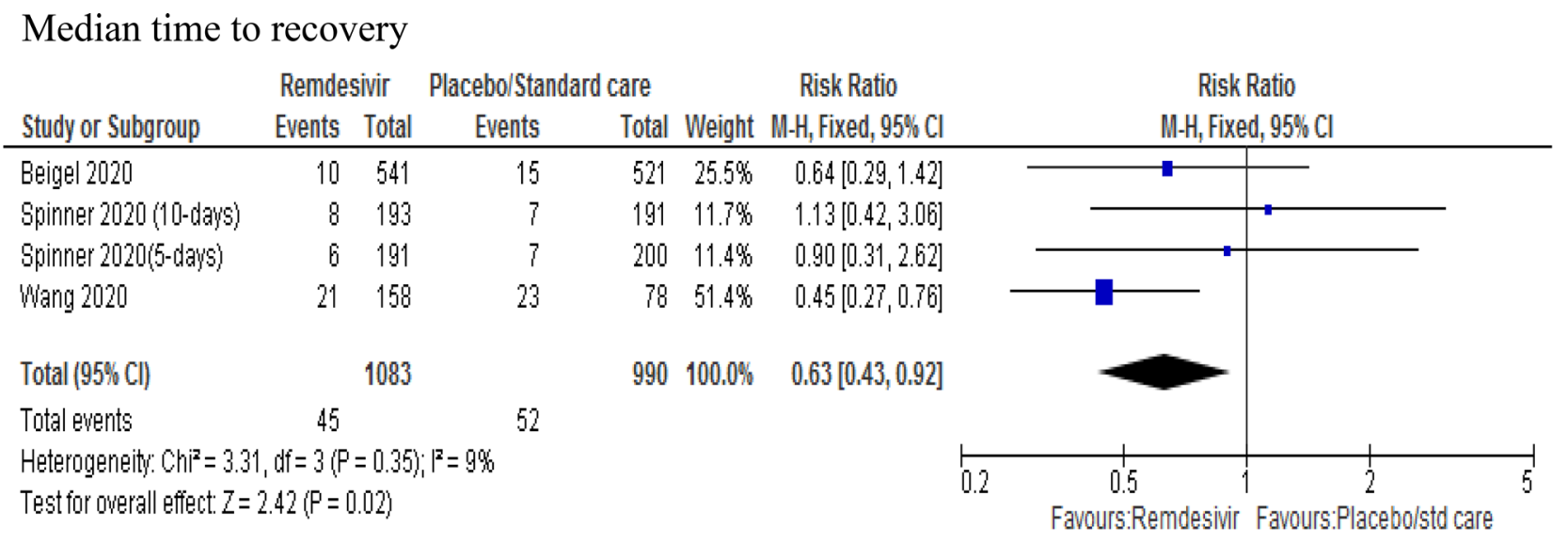

Median time to discharge

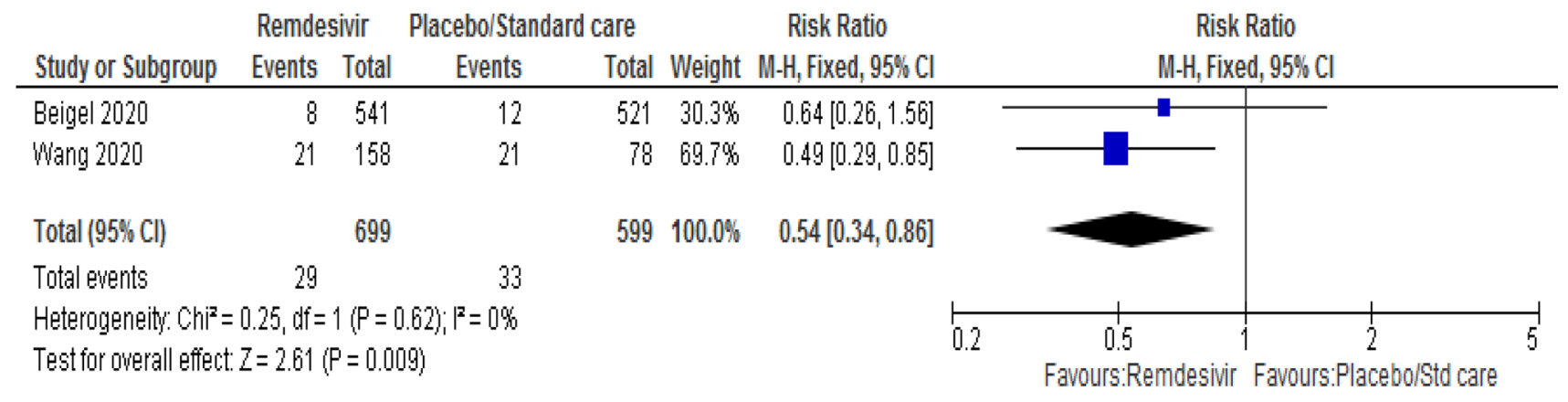

Fig. 5 Forest plot of median time to recovery and discharge

95\% CI 0.77-0.90) than patients treated with placebo. Our pooled data suggest that treatment with remdesivir may have prevented complications and serious respiratory failure, as shown by lower requirement for supplemental oxygen; a lower proportion of patients needing higher levels of respiratory support and a shorter subsequent duration of mechanical ventilation or ECMO. This was supported by a systematic review and meta-analysis of 3 RCTs that consisted of 1691 patients and revealed that the odds for mechanical ventilation was significantly lower in the remdesivir group than the control group (OR 0.48; 95\% CI 0.34; 0.69) [21]. Overall, the findings suggest that treatment with remdesivir is associated with the reduction in the disease burden and use of scarce health care resources during this pandemic.

The present review revealed that the remdesivir group had lower risk of mortality (RR $0.61 ; 95 \%$ CI $0.46-0.79$, $p=0.003$ ) than the placebo-treated group on day 14 . A metaanalysis of four studies [34] revealed that remdesivir-treated patients had a lower risk of mortality than placebo (OR 0.61; $95 \%$ CI $0.45-0.82 ; p=0.001)$ on day 14 . Likewise, a systematic review of four RCTs for an American College of Physicians Practice Points [26] also claimed that remdesivir may reduce mortality with absolute risk difference (ARD) ranging from -4 to $1 \%$ compared to the placebo. On day 28 , however, there was no significant difference in mortality between the remdesivir and placebo groups. This is in line with a systematic review and meta-analysis conducted by Reddy-Vegivinti et al. [21] and Okali GN et al. [22], which showed that remdesivir has no effect in reducing day-28 mortality in patients with COVID-19 possibly due to reduced antiviral activity. Early initiation of remdesivir was associated with a mortality benefit on day 14 due to reduction in time to clinical improvement, highlighting the need for appropriately timed remdesivir treatment in moderate-tosevere COVID-19. Further research is needed to clarify the diminishing effect of remdesivir therapy in reducing mortality over time for patients with COVID- 19 .

A pooled analysis of four studies revealed that serious adverse effects in patients treated with remdesivir were significantly lower than the placebo group, with an absolute risk difference of $6 \%(\mathrm{RD}-0.06 ; 95 \% \mathrm{CI}-0.09$ to -0.03 ). Specifically, treatment with remdesivir was associated with a $26 \%$ lower risk rate of grade 3 (severe) and grade 4 (lifethreatening) adverse effects (RR 0.74; 95\% CI 57-97). 
Fig. 6 Forest plot of safety outcomes of remdesivir

Number of patients with serious adverse events

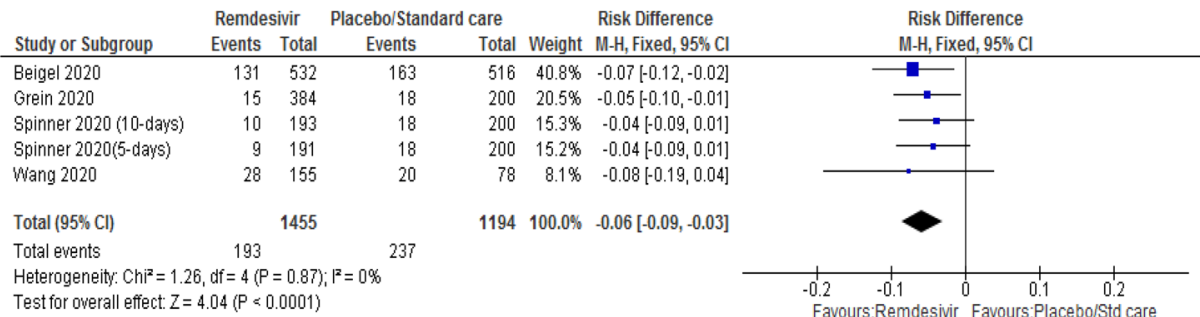

Number of patients who discontinued treatment due to adverse events

\begin{tabular}{|c|c|c|c|c|c|c|c|c|}
\hline \multirow[b]{2}{*}{ Study or Subgroup } & \multicolumn{2}{|c|}{ Remdesivir } & \multicolumn{2}{|c|}{ Placebo/Standard care } & \multirow[b]{2}{*}{ Weight } & \multirow{2}{*}{$\begin{array}{l}\text { Risk Difference } \\
\text { M-H, Fixed, } 95 \% \mathrm{Cl}\end{array}$} & \multirow{2}{*}{$\begin{array}{c}\text { Risk Difference } \\
\text { M- } \mathrm{H}, \text { Fixed, } 95 \% \mathrm{Cl}\end{array}$} & \\
\hline & Events & Total & Events & Total & & & & \\
\hline Beigel 2020 & 57 & 532 & 77 & 516 & $51.4 \%$ & $-0.04[-0.08,-0.00]$ & $\nabla$ & \\
\hline Spinner 2020 (10-days) & 8 & 193 & 0 & 200 & $19.3 \%$ & $0.04[0.01,0.07]$ & - & \\
\hline Spinner 2020(5-days) & 4 & 191 & 0 & 200 & $19.2 \%$ & $0.02[-0.00,0.04]$ & - & \\
\hline Wang 2020 & 18 & 155 & 4 & 78 & $10.2 \%$ & $0.06[-0.01,0.14]$ & - & \\
\hline Total $(95 \% \mathrm{Cl})$ & & 1071 & & 994 & $100.0 \%$ & $-0.00[-0.03,0.02]$ & 1 & \\
\hline Total events & 87 & & 81 & & & & & \\
\hline $\begin{array}{l}\text { Heterogeneity: } \mathrm{Ch}^{2}=20 \text {. } \\
\text { Test for overall effect: } Z=\end{array}$ & $\begin{array}{l}9, d f=3(P \\
.26(P=0\end{array}$ & $\begin{array}{l}P=0.00 \\
1.80\end{array}$ & $02 ; ;\left.\right|^{2}=85 \%$ & & & & 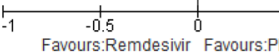 & $\begin{array}{c}0.5 \\
\text { Placebo }\end{array}$ \\
\hline
\end{tabular}

Number of patients with grade 3 (severe) and grade 4 (life-threatening) ADEs

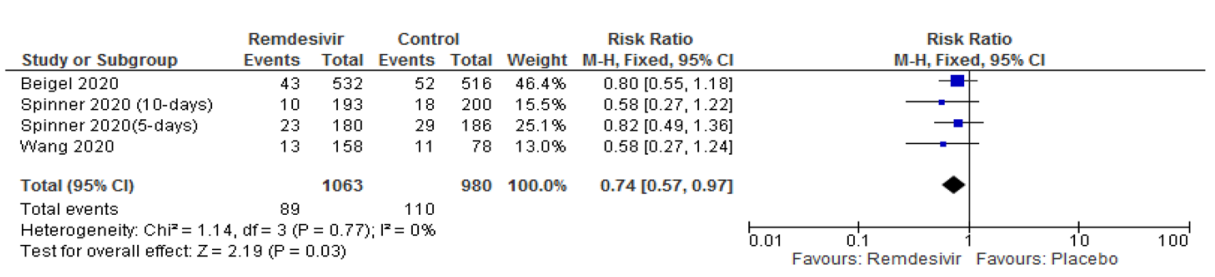

Number of patients with elevated AST level

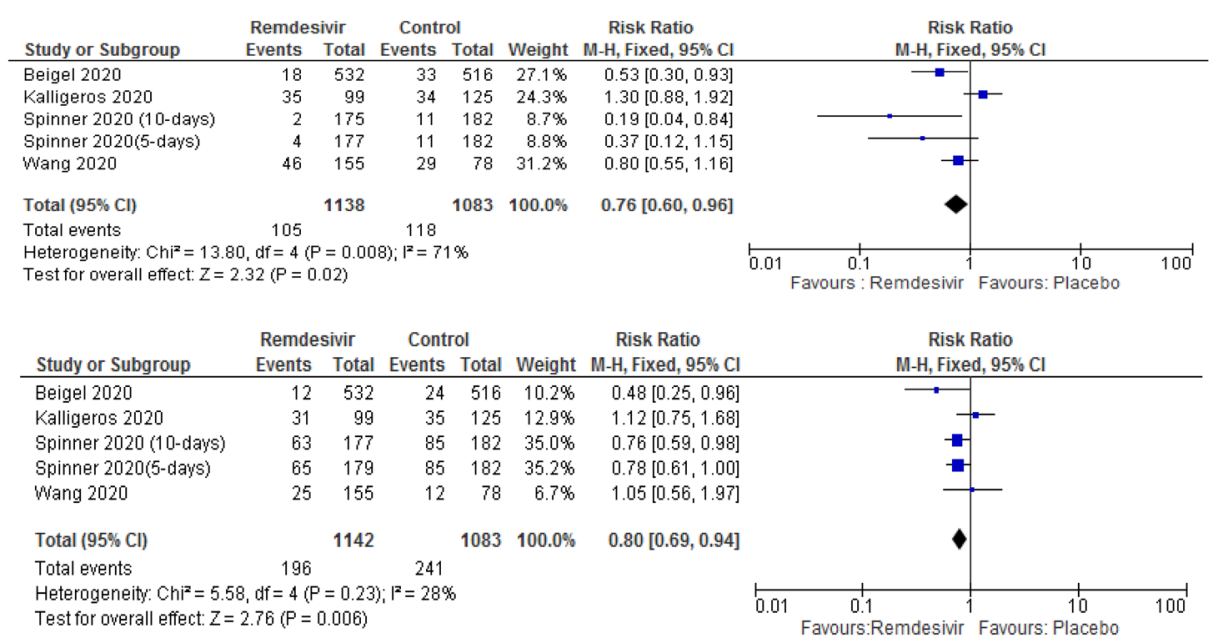

Number of patients with decreased level of serum creatinine level

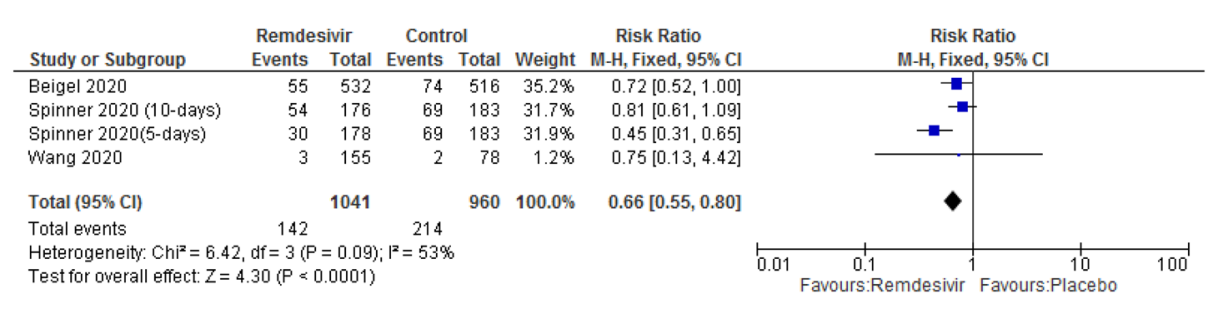


Additionally, treatment with remdesivir was associated with a lower risk of AST and ALT elevation than placebo with a relative risk of $42 \%$ (RR $0.58 ; 95 \%$ CI $0.43-0.79$ ) and $20 \%$ (RR 0.80; $95 \%$ CI 0.69-0.94; $p=0.006$ ), respectively. The relative risk of serum creatinine elevation (RR 0.66; 95\% CI $0.55-0.80 ; p<0.001)$ was also lower in the remdesivir-treated group than the placebo. A systematic review and subgroup analysis by $\mathrm{Xu}$ et al. [35] revealed that remdesivir did not increase the incidence of acute kidney injury in patients with COVID-19. Three RCTs [9-11] independently reported that a similar proportion of adverse effects occurred in the remdesivir and placebo groups. Spinner et al. [13] reported a higher proportion of nausea, hypokalaemia, and headache among remdesivir-treated patients. Likewise, Montastruc et al. [36] (with no comparator group) reported an increased risk of hepatic disorders with remdesivir treatment. In the study by Wang et al. [10], more patients in the remdesivir group than the placebo (12\% versus $5 \%)$ discontinued the drug because of serious adverse effects such as respiratory failure or acute respiratory distress syndrome. This review, however, revealed that there was no significant difference between remdesivir and placebo groups in terminating treatment due to adverse effects (RD: $-0.00,95 \% \mathrm{CI}$ : -0.03-0.02). Although there were conflicting results, remdesivir was well tolerated compared with the placebo yet available evidence from clinical studies support the need to conduct close hepatic and renal monitoring in patients receiving remdesivir.

\section{Strengths and limitations}

This systematic review and meta-analysis pooled results from 3686 patients in 7 completed studies. It provided a comprehensive meta-analysis output on clinical, mortality and safety benefits of remdesivir over standard care or placebo. Compared with available reviews [18, 19, 34], our study included a greater number of studies and patients and determined more positive and robust evidences regarding remdesivir-associated mortality and safety. Despite these strengths, our review has some limitations. There were openlabel designs in some studies included in the meta-analysis, which might potentially have led to biases in both patient care and reporting of data. There was lack of validation of clinical efficacy of remdesivir and the placebo with the viral loads of the patients in both groups. The clinical efficacy of remdesivir was assessed by six, seven and eight-category ordinal scale, which might not necessarily indicate the biological mechanisms that are required to interpret the clinical efficacy of it. There are differences in definitions of severity of COVID-19 cases, ordinal scales and clinical recoveries between studies, thus, our pooled results should be interpreted carefully. There was heterogeneity in the age groups among the trials in which two studies recruited patients aged $\geq 12$ years, while the rest recruited patients aged 18 years and over. The risk of publication bias could not be assessed due to the low number of the included studies.

\section{Conclusions}

Although there were conflicting results, remdesivir could still be effective in early clinical improvement; reduction of early mortality and avoiding high-flow supplemental oxygen and invasive mechanical ventilation among hospitalised COVID-19 patients. Remdesivir was also well tolerated without significant serious adverse effects compared to placebo, yet available evidence from clinical studies support the need to conduct close monitoring. Further research is needed to clarify the declining effect of treatment with remdesivir in reducing mortality over time for patients with COVID-19.

Supplementary Information The online version contains supplementary material available at https://doi.org/10.1007/s15010-021-01671-0.

Acknowledgements Authors would like to acknowledge Luke Bereznicki and Leanne Chalmers for their substantial contribution in critically revising this manuscript for an important academic content.

Author contributions MA contributed to the study conception and design, literature search and study selection, data extraction, data synthesis, manuscript writing and assessment of risk of bias. GP supervised the progress of the work, assisted in solving the discrepancies encountered in the article review process and interpreting the results and critically revising the manuscript. MM worked on literature search and study selection, data extraction, assessment of risk of bias and critically revising the manuscript. All authors read and approved the final manuscript.

Funding Not applicable.

\section{Declarations}

Conflict of interest The authors declare that they have no conflict of interest.

Ethical approval Not applicable.

\section{References}

1. World Health Organization. Coronavirus Disease-19 (COVID-19) Dashboard. 2021. https://covid19.who.int/. Accessed 1 Feb 2021.

2. World Health Organization. Coronavirus disease-2019 (COVID19) Situation Report. 2021. https://www.who.int/emergencies/ diseases/novel-coronavirus-2019/situation-reports. Accessed 3 Feb 2021.

3. World Health Organization. Events as they happen. Rolling updates on coronavirus disease-19 (COVID-19). Geneva: World Health Organization; 2020. 
4. Pak A, Adegboye OA, Adekunle AI, Rahman KM, McBryde ES, Eisen DP. Economic consequences of the COVID-19 outbreak: the need for epidemic preparedness. Front Public Health. 2020. https://doi.org/10.3389/fpubh.2020.00241.

5. Grein J, Ohmagari N, Shin D, Diaz G, Asperges E, Castagna A, et al. Compassionate use of remdesivir for patients with severe Covid-19. N Engl J Med. 2020;382:2327-36. https://doi.org/10. 1056/NEJMoa2007016(1533-4406(Electronic)).

6. Cao B, Wang Y, Wen D, Liu W, Wang J, Fan G, et al. A trial of lopinavir-ritonavir in adults hospitalized with severe Covid-19. N Engl J Med. 2020;382:1787-99 (1533-4406 (Electronic)).

7. Saber-Ayad MA-O, Saleh MA-O, Abu-Gharbieh EA-O. The Rationale for potential pharmacotherapy of COVID-19. Pharmaceuticals (Basel). 2020;13:96 (1424-8247 (Print)).

8. National Institute of Health. COVID-19 Treatment Guidelines. 2021. https://www.covid19treatmentguidelines.nih.gov/whatsnew/. Accessed 29 Jan 2021.

9. Beigel JA-O, Tomashek KM, Dodd LE, Mehta AK, Zingman BS, Kalil AA-O, et al. Remdesivir for the treatment of Covid19: final report. N Engl J Med. 2020;383:1813-26 (1533-4406 (Electronic)).

10. Wang Y, Zhang D, Du G, Du R, Zhao J, Jin Y, et al. Remdesivir in adults with severe COVID-19: a randomised, double-blind, placebo-controlled, multicentre trial. Lancet. 2020;395:1694 (1474-547X (Electronic)).

11. Goldman JA-O, Lye DCB, Hui DS, Marks KM, Bruno R, Montejano R, et al. Remdesivir for 5 or 10 days in patients with severe Covid-19. N Engl J Med. 2020;383:1827-37 (1533-4406 (Electronic)).

12. Gillenwater $\mathrm{S}$, Rahaghi $\mathrm{F}$, Hadeh $\mathrm{A}$. Remdesivir for the treatment of Covid-19: preliminary report. N Engl J Med. 2020;383:992-4 (1533-4406 (Electronic)).

13. Spinner CD, Gottlieb RL, Criner GJ, Arribas López JR, Cattelan AM, Soriano Viladomiu A, et al. Effect of remdesivir vs standard care on clinical status at 11 days in patients with moderate COVID-19: a randomized clinical trial. J Am Med Assoc. 2020;324:1048-57 (1538-3598 (Electronic)).

14. Food and Drug Administration. Coronavirus Disease-19 (COVID19) update: FDA issues emergency use authorization for potential COVID-19 treatment. 2021. https://www.fda.gov/newsevents/pressannouncements/coronavirus-covid-19-update-fdais sues-emergency-use-authorization-potentialcovid-19-treatment. Accessed 1 Mar 2021.

15. European Medicines Agency. First COVID-19 treatment recommended for EU authorization. 2021. https://www.ema.europa.eu/ en/news/firstcovid-19-treatment-recommended-euauthorisation. Accessed 15 Feb 2021.

16. Davies M, Osborne V, Lane S, Roy D, Dhanda S, Evans A, et al. Remdesivir in treatment of COVID-19: a systematic benefit-risk assessment. Drug Saf. 2020;43:645-56.

17. Shrestha DB, Budhathoki P, Syed NI, Rawal E, Raut S, Khadka S. Remdesivir: a potential game-changer or just a myth? A systematic review and meta-analysis. Life Sci. 2021;264: 118663.

18. Elsawah HA-O, Elsokary MA, Abdallah MS, ElShafie AH. Efficacy and safety of remdesivir in hospitalized Covid-19 patients: systematic review and meta-analysis including network metaanalysis. Rev Med Virol. 2020;31: e2187.

19. Piscoya A, Ng-Sueng LF, Parra del Riego A, Cerna-Viacava R, Pasupuleti V, Roman YM, et al. Efficacy and harms of remdesivir for the treatment of COVID-19: a systematic review and metaanalysis. PLoS ONE. 2020;15: e0243705.

20. Al-Abdouh A, Bizanti A, Barbarawi M, Jabri A, Kumar A, Fashanu OE, et al. Remdesivir for the treatment of COVID-19: a systematic review and meta-analysis of randomized controlled trials. Contemp Clin Trials. 2021;101: 106272.

21. Reddy Vegivinti CT, Pederson JM, Saravu K, Gupta N, Barrett A, Davis AR, et al. Remdesivir therapy in patients with COVID-19: a systematic review and meta-analysis of randomized controlled trials. Ann Med Surg. 2012;2021:43-8.

22. Okoli GN, Rabbani R, Copstein L, Al-Juboori A, Askin N, AbouSetta AM. Remdesivir for coronavirus disease 2019 (COVID-19): a systematic review with meta-analysis and trial sequential analysis of randomized controlled trials. Infect Dis. 2021. https://doi. org/10.1080/23744235.2021.1923799.

23. Enoki Y, Igarashi Y, Watabe Y, Honma K, Suzuki Y, Hayashi Y, et al. Remdesivir for the treatment of coronavirus COVID-19: a meta-analysis of randomised controlled trials. J Glob Antimicrob Resist. 2020;24:81-2.

24. Yokoyama Y, Briasoulis A, Takagi H, Kuno T. Effect of remdesivir on patients with COVID-19: a network meta-analysis of randomized control trials. Virus Res. 2020;288: 198137.

25. Jiang Y, Chen D, Cai D, Yi Y, Jiang S. Effectiveness of remdesivir for the treatment of hospitalized COVID-19 persons: a network meta-analysis. J Med Virol. 2021;93:1171-4.

26. Rezagholizadeh A, Khiali S, Sarbakhsh P, Entezari-Maleki T. Remdesivir for treatment of COVID-19; an updated systematic review and meta-analysis. Eur J Pharmacol. 2021;897: 173926.

27. Wilt TJ, Kaka AS, MacDonald R, Greer N, Obley A, Duan-Porter W. Remdesivir for adults with COVID-19: a living systematic review for an american college of physicians practice points. Ann Intern Med. 2020. https://doi.org/10.7326/M20-5752.

28. Higgins JP, Altman DG, Gøtzsche PC, Jüni P, Moher D, Oxman $\mathrm{AD}$, et al. The Cochrane Collaboration's tool for assessing risk of bias in randomised trials. Br Med J. 2011;343: d5928.

29. Sterne JA, Hernán MA, Reeves BC, Savović J, Berkman ND, Viswanathan M, et al. ROBINS-I: a tool for assessing risk of bias in non-randomised studies of interventions. BMJ. 2016;355: i4919. https://doi.org/10.1136/bmj.i4919 (1756-1833(Electronic)).

30. Sterne JAC, Sutton AJ, Ioannidis JPA, Terrin N, Jones DR, Lau $\mathrm{J}$, et al. Recommendations for examining and interpreting funnel plot asymmetry in meta-analyses of randomised controlled trials. Br Med J. 2011;343: d4002.

31. Julian PTH, Sally G. Cochrane handbook for systematic reviews of interventions: The Cochrane Collaboration. Version 5.1.0. 2011. https://handbook-5-1.cochrane.org/. Accessed 10 Jan 2021.

32. Olender SA, Perez KK, Go AS, Balani B, Price-Haywood EG, Shah NS, et al. Remdesivir for severe COVID-19 versus a cohort receiving standard of care. Clin Infect Dis. 2020. https://doi.org/ 10.1093/cid/ciaa1041 (1537-6591 (Electronic)).

33. Kalligeros M, Tashima KT, Mylona EK, Rybak N, Flanigan TP, Farmakiotis D, et al. Remdesivir use compared with supportive care in hospitalized patients with severe COVID-19: a singlecenter experience. Open Forum Infect Dis. 2020;7: ofaa319 (2328-8957 (Print)).

34. Sarfraz A, Sarfraz Z, Sanchez-Gonzalez M, Michel J, Michel G, Frontela $\mathrm{O}$, et al. Randomized controlled trials of remdesivir in hospitalized COVID-19 patients: a systematic review and metaanalysis. Medrxiv. 2020;6:672.

35. Xu Z, Tang Y, Huang Q, Fu S, Li X, Lin B, et al. Systematic review and subgroup analysis of the incidence of acute kidney injury (AKI) in patients with COVID-19. BMC Nephrol. $2021 ; 22: 52$.

36. Montastruc F, Thuriot S, Durrieu G. Hepatic disorders with the use of remdesivir for Coronavirus 2019. Clin Gastroenterol Hepatol. 2020;18:2835-6. 